\title{
CONTROLLED AND ADAPTIVE MESH ZIPPERING
}

\author{
Stefano Marras \\ University of Cagliari, Italy. \\ stefano.marras@unica.it \\ Fabio Ganovelli Paolo Cignoni \\ ISTI-CNR, Italy \\ Riccardo Scateni \\ University of Cagliari, Italy. \\ Roberto Scopigno \\ ISTI-CNR, Italy
}

Keywords: Zippering, Stitching, 3D scanning.

\begin{abstract}
Merging meshes is a recurrent need in geometry modeling and it is a critical step in the 3D acquisition pipeline, where it is used for building a single mesh from several range scans. A pioneering simple and effective solution to merging is represented by the Zippering algorithm (Turk and Levoy, 1994), which consists of simply stitching the meshes together along their borders. In this paper we propose a new extended version of the zippering algorithm that enables the user to control the resulting mesh by introducing quality criteria in the selection of redundant data, and allows to zip together meshes with different granularity by an ad hoc refinement algorithm.
\end{abstract}

\section{INTRODUCTION AND PREVIOUS WORK}

In recent years, the technology for digitizing real $3 \mathrm{D}$ objects has greatly improved and became more accessible to the general audience. While only ten years ago the acquisition of the Michelangelo's David (Pereira et al., 2000) required 22 people for 30 days and the prototypical device sampled $14 k$ points per second, nowadays it is possible to acquire a similar device for less than $3000 \$$ (NextEngine, ). However, notwithstanding the impressive improvement and diffusion of the hardware, the 3D scanning software pipeline is substantially unchanged: once the single range maps have been acquired with the device, they must be aligned in the same reference system, then merged in a single triangle mesh. After these steps others may follow for including colors and/or for optimizing the resulting mesh for rendering. Over the years each and every step has been refined in several regards, and nowadays we can safely state that the $3 \mathrm{D}$ acquisition pipeline is a stable process. Nonetheless, further improvements still can be done for example in terms of computation time, which is what we do in this work, focus- ing on the process of merging aligned range maps.

A range map is an image where each pixel stores a depth value, which means that information contained in a range map is a set of points in $3 \mathrm{D}$ space. Given the regular distribution of the samples in the image space, it is possible to triangulate them to obtain a triangle mesh for each range map.

An early mesh merging approach, called zippering (Turk and Levoy, 1994), consists in stitching together the pairs of triangulations that overlap in 3D space by eliminating the redundant faces in the overlapping regions and then adjusting the mesh connectivity locally. Other approaches work by triangulating union of the point sets, like the Ball Pivoting (Bernardini et al., 1999), which consists of rolling an imaginary ball on the point sets and creating a triangle for each triplet of points supporting the ball. This approach is strictly connected to the idea of $\alpha$ shapes (Edelsbrunner and Mücke, 1994), which may be thought as a ball pivoting where the ball may appear simultaneously everywhere outside the scanned volume. In other approaches, like in (Duan and Qin, 2003), active contours are used to deform a mesh to fit the sample points. Note 
that in these cases the vertices of the final mesh do not coincide with the initial samples. In fact, the reconstruction of the final representation is also a way to filter the input data to reduce noise and discard outliers.

Imperfections and noise produced by the scanning devices is one of the reasons for the success of volumetric methods, which convert the range maps in a volumetric domain (a discrete distance field), well suited for filtering and merging operations. The Space Carving proposed in (Curless, 1999) uses range maps and line of sight of the scanner to determine a configuration of the empty voxels consistent with all the range images. Many of the improvements to volumetric methods consist of enhanced methods for estimating distances and normals of the surface. The method proposed in (Hoppe et al., 1992) uses local estimation of tangent planes to obtain a signed distance function. More recent approaches uses MLS local approximations of the surface to address continuity of the distance function (Alexa et al., 2001; Shen et al., 2005) which may be then visualized by sampling the isosurface with points, using contouring techniques (Fiorin et al., 2007) to build a polygon mesh or ray tracing it at rendering time (Guennebaud and Gross, 2007). A quadratic local approximation scheme has been combined with a hierarchical space subdivision that allows the reconstructions of the surface for large number of points in (Ohtake et al., 2003). In the same flavor, the Poisson Surface Reconstruction algorithm (Kazhdan et al., 2006) formulates the problem of defining the surface from a set of point as a Poisson problem, for which a least square solution can be efficiently found at different scales.

The revisitation of the mesh zippering as a mesh merging algorithm is mainly motivated by the fact that, thanks to the improvement of both the software and the hardware involved in the 3D scanning pipeline, the quality of acquisition and range maps alignment may allow to zip them together and to obtain the same result as with more costly and sophisticated methods.

\section{ALGORITHM OVERVIEW}

Our zippering algorithm consists of the same steps as the original version presented in (Turk and Levoy, 1994), that we summarizes in the following. Given two meshes $A$ and $B$ that partially overlap, the zippering algorithm proceeds in three
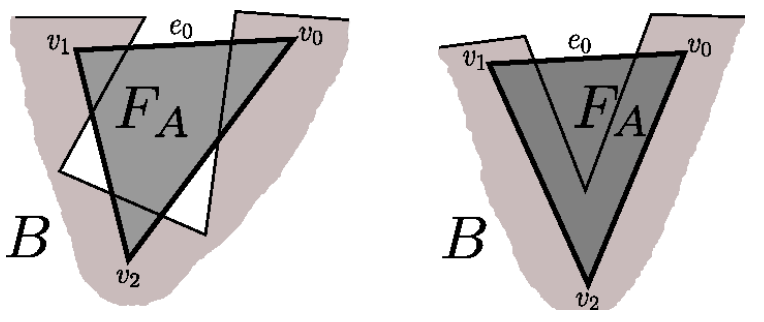

Figure 1: False Positive

phases:

- Border Erosion. Remove faces from the border of the patches to minimize data redundancy.

- Clip a patch against the other. The border of one of the two range maps is projected on the other range map, the faces intersected by projection of $A$ 's border are retriangulated in order to create a new consistent connectivity.

- Cleaning. Improve the quality of the mesh in the region when retriangulation occurred.

These phases are revisited to extend the zippering to support a user-defined criterion to eliminate the data redundancy and to robustly support the zippering of patches with very different resolution.

\subsection{Border Erosion}

In the original zippering algorithm (Turk and Levoy, 1994) a triangular face is said to be redundant if its 3 vertices project on the surface of the other patch. As it is shown in Figure 1 this process may easily create holes of roughly the size of the face, that will be eventually triangulated after the zippering process. However there are situations in which we cannot make the assumption that range maps to be zippered are similar in terms of size and number of polygons, for example because more than one device is used to scan the surface and the two produce range maps with different granularity. This is quite common in scanning Cultural Heritage artifacts where there are portions of the surface that cannot be accessed with the device used and are subsequently covered with a different one (Dellepiane et al., 2009).

Therefore we compute the distance between a face of a patch and the other patch by using a uniform sampling of the face, in order to guarantee a bounded size of the holes that we may create. We say that a point projects on the 


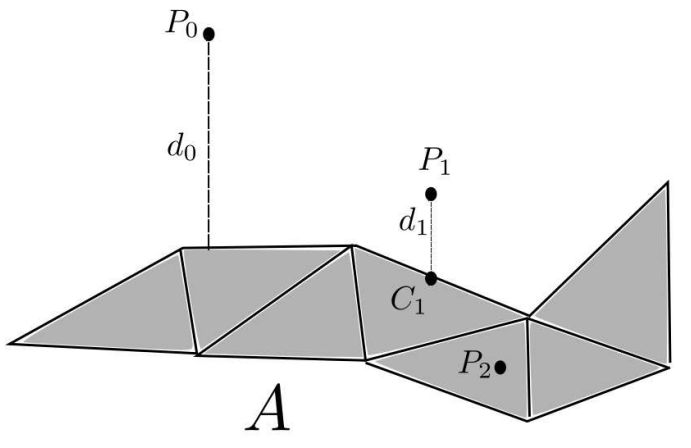

Figure 2: Three points and a patch $A$ : distance $d_{0}$ between $P_{0}$ and $A$ is greater than a given threshold $\varepsilon$, so $P_{0}$ does not project on $A$; distance $d_{1}$ between $P_{1}$ and $A$ is lower than $\varepsilon$, but the closest point $C_{1}$ is on a border edge of $A$, so also $P_{1}$ does not project on $A$; finally, $P_{2}$ project on $A$, since its distance from $A$ is lower than $\varepsilon$ and closest point does not lie on border of $A$.

patch if it is closer than a given threshold to the patch and the closest point is not on a border edge (see Figure 2). Our algorithm classifies a face of patch $\mathrm{A}$ as redundant if all the samples project on the patch $B$ (and vice versa).

The meaning of eliminating redundant faces is to redefine the border of the patches, in other terms to establish a frontier to divide the region where faces of the mesh $\mathrm{A}$ are taken as valid data from the region where faces of the mesh $\mathrm{B}$ are taken. Figure 3 shows three different frontiers for the same pair of range maps. In the original algorithm a single patch is chosen as the one containing redundant information and therefore only its faces are possibly redundant. Conversely, our algorithm chooses to test redundant faces on the base of a quality value that we may use, for example, to preserve faces with lower estimated acquisition error, or with a better aspect ratio.

Figure 4 sketches the erosion algorithm. The queue $Q$ stores the faces on the border of the two patches in ascending order of quality; the face with lowest quality will be the first one of the queue. Starting from the first face in the queue, we check all faces, testing redundancy. Each face is tested once, and then it is removed from the queue; if the face is redundant, then it will be also removed from the original mesh, and its neighbors will be added to $Q$. The process stops when $Q$ is empty, and all the faces involved in the process have been tested.
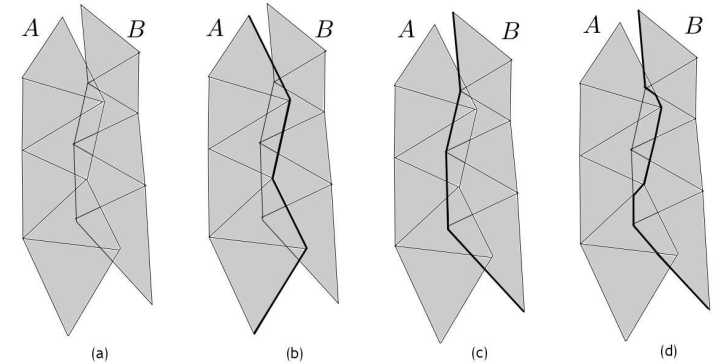

Figure 3: Two patches (a), labeled as $A$ and $B$, and possible frontiers between them; the frontier can be made of border faces from A (b) or B (c), or it can be mixed with part of border from $\mathrm{A}$ and part from B (d).

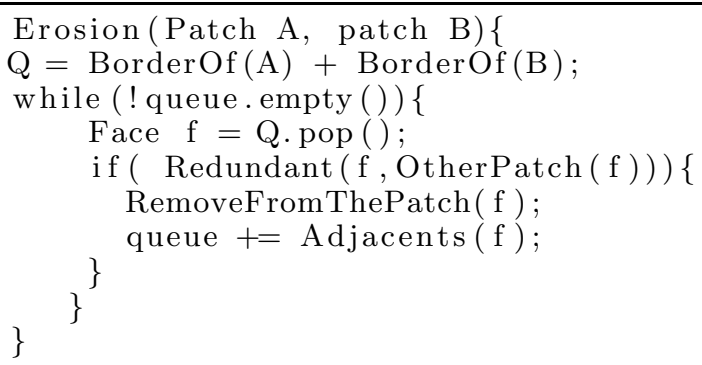

Figure 4: The erosion algorithm selects the lowest priority face until there is redundancy of data.

\subsection{Clipping}

At this point, the redundant faces have been removed from $A$ and $B$ and we are in a situation where there is an overlap between the two meshes so that every face on the border of a patch is only partially overlapping the other patch.

Our clipping algorithm consist of two steps:

1. refine the faces on the border of the patch $A$ until no border edge projects on more than one face of $\mathrm{B}$

2. remove from each face of $B$ the part covered by a projection and retriangulate the remain.

\subsubsection{Refinement}

The first step if carried out as follows. We keep a queue $B_{A}$ of faces of $\mathrm{A}$ to be processed and initialize it with the border faces of $A$. Then the following steps are applied until the queue is empty.

Remove a face $F_{A}$ from $B_{A}$, project its two border vertices, named $v_{0}$ and $v_{1}$, on the surface of $B$. Let $F_{0}$ and $F_{1}$ be the faces of B where projections of $v_{0}$ and $v_{1}$ lie, and let $e_{0}$ the border 
edge between $v_{0}$ and $v_{1}$. Then one of the followings holds:

- $F_{0}$ and $F_{1}$ are the same face, in this case, the projection of $e_{0}$ lies completely inside the face, we associate the face $F_{A}$ with $F_{0}$ (see Figure 5.(a)).

- $F_{0}$ is adjacent to $F_{1}$, that is, they share an edge; we name this edge $e_{S}$, in this case we create a vertex on the edge $e_{S}$ positioned at the closest point of $e_{S}$ to $e_{0}$ and replace the face $F_{A}$ with two faces as shown in Figure 5.(b), which are inserted in $B_{A}$.

- $F_{0}$ is not adjacent to $F_{1}$, in this case we simply split the face $F_{A}$ along the middle point of the border edge and insert the resulting faces in $B_{A}$ (see Figure 5.(c).

- One of the two vertices does not project on B (see Figure 6.(a)), in this case we simply consider the non projecting vertex, say it is $v_{0}$ as projecting in an ideal face of patch $\mathrm{B}$ passing through $v_{0}$ and process the faces as explained in the previous cases.

- None of the vertices project, in which case there is no action to take (see Figure 6.(b)).

In other terms, we refine the faces until the first case is verified (see Figure 5.(c)). Note that the last two cases always happen when only a portion of one patch overlaps the others, while may not be encountered when using a patch to close a hole.

\subsubsection{Removal and Retriangulation}

At this point each of the projecting faces of A will be associated with a face of $\mathrm{B}$. What we need to do is to decompose each face of $\mathrm{B}$ in a part clipped by the projection of the faces of $\mathrm{A}$ and a part to be retriangulated accordingly to such projections. As shown in Figure 7 for each face $F_{B}$ to clip we will have one or more polylines with ending vertices exactly on the edges of $F_{B}$, which allow to separate the face in non-clipped and clipped part. Then we only need to discard the clipped parts and to retriangulate the remaining polygon. Note that in rare situations we can also have more than one remaining polygon for the same face (see Figure 7.(c)).

\subsection{Cleaning}

The quality of the faces is an important part of any reconstruction techniques. In this sense the zippering bears a clear disadvantage since it uses a constrained triangulation. On the other hand,

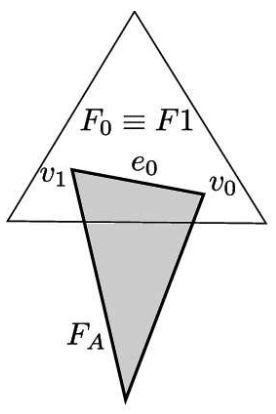

(a)

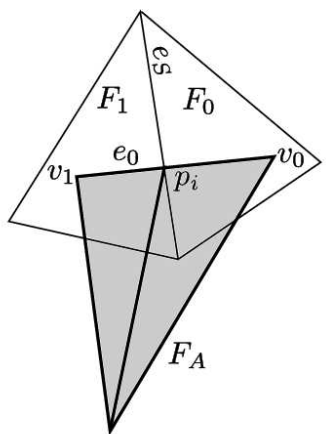

(b)

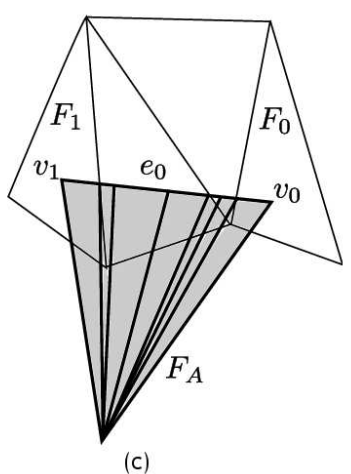

Figure 5: (a) projection of both vertices lie in one face; (b) in two adjacent faces. (c) projections lie on non adjacent faces: the border face is recursively split.

we know exactly the region of the resulting mesh where the quality of the faces tends to be poor and may selectively filter those parts. Furthermore, we know that the poorly shaped faces are mostly the result of almost planar subdivision (during the refinement step) or planar retriangulations (the final part of clipping).

\section{EXPERIMENTAL RESULTS}

In this section, we present some results of the experiments conducted. For each result we also gave information about time consumed by each stage of the algorithm. All of the experiments were performed on a Inter Quad-Core Q9550 $2.83 \mathrm{GHz}$ equipped with a NVidia GeForce GTX 260 and 4,00 GB of on-board memory. Our algorithm has been developed as a plugin for the MeshLab system (Cignoni et al., 2008). Figure 9 shows a model of a car with a large missing part and the patch that covers the missing part (top row). We applied our algorithm with three dif- 


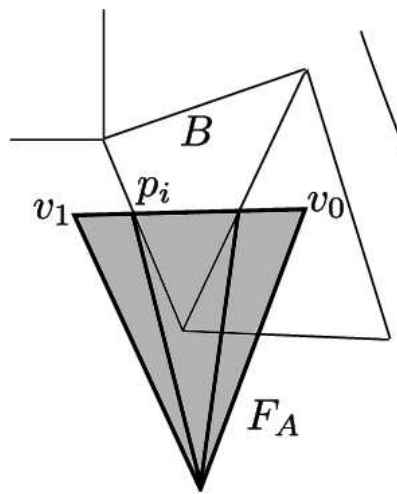

(a)

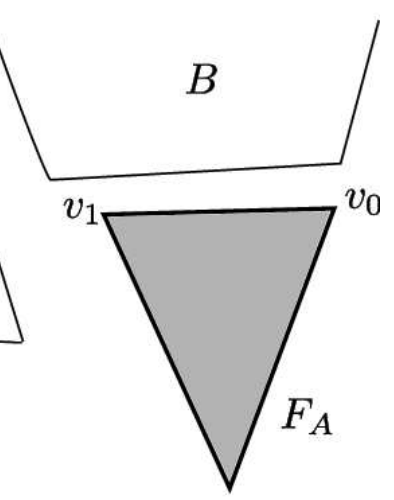

(b)
Figure 6: Two additional case: vertex $v_{1}$ is not projectable on mesh, so edge is split on point $p_{i}$, then only segment from $v_{0}$ to $p_{i}$ is projected on $B$; both $v_{0}$ and $v_{1}$ are not projectable, so $F_{A}$ will not be modified.

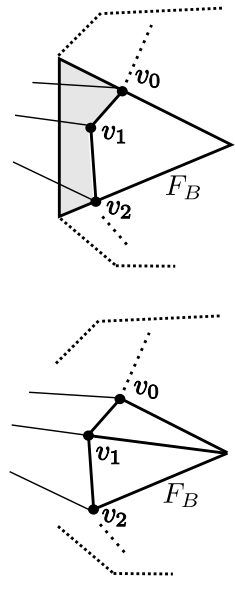

(a)
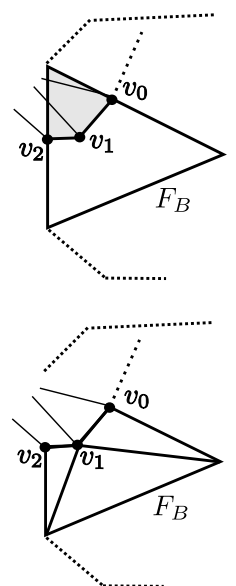

(b)
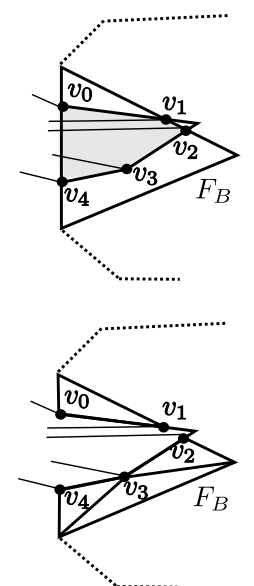

(c)
Figure 7: Examples of face clipping. The polygon shaded in gray is to be removed, the rest of face $F_{B}$ will be retriangulated.

ferent criteria: by preserving the faces of the model, by preserving the faces of the patch and by weighting the faces on the base of their distance from the border, so obtaining a clipping frontier in the middle of the previous two. Table 1 reports the timing for the three cases.

The most interesting experiment is the comparison with the Poisson Surface Reconstruction Algorithm (Kazhdan et al., 2006) to merge two range maps with $2.5 \mathrm{M}$ triangles each. The range maps, obtained with a Breukmann Smartscan laser scanner1 (Breuckmann, ), are very dense (100 samples per millimeter) and very well aligned. Figure 10 shows two range maps merged using PSR (left) and the result or our zippering algorithm (right). The bottom of the figure shows the Hausdorff distance between the two results mapped as false color on the mesh. As expected, the result is essentially the same and the sewing regions are not noticeable. However, our zippering algorithm took $1 \mathrm{~m} 27 \mathrm{~s}$ seconds to complete against the $6 \mathrm{~m} 16 \mathrm{~s}$ of the Poisson Surface Reconstruction. Note that the PSR is done with the original source code provided by the authors and in the same hardware setup.

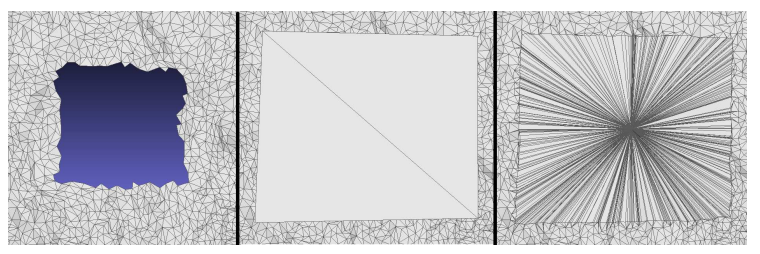

Figure 8: Test case. A hole in a dense mesh (left) is covered with with a quad (center) and the algorithm produces a conformal mesh.

\section{CONCLUSION}

We presented an improved version of the zippering algorithm for merging meshes which extends the original version by enabling enhanced control over the redundancy of data and supporting merging of meshes with very different granularity. In the examples above, we presented results of merging of two different mesh, but the procedure can be easily extended in order to manage more than two meshes, e.g. two or more patches covering a single hole, or two or more range maps partially overlapping. Our experiments shown that with modern range scanners and thanks to the significant improvements made to the scanning pipeline since the dawn of $3 \mathrm{D}$ scanning, mesh zippering is back as a viable and efficient solution to merging.

\section{Aknowledgements}

The research leading to these results has received funding from the EG $7 \mathrm{FP}$ IP "3DCOFORM" project (2008-2012, n. 231809). 


\begin{tabular}{|l|l|l|l|}
\hline Criteria & Erosion & Clipping & Cleaning \\
\hline Preserving model's face & $938 \mathrm{~ms}$ & $1018 \mathrm{~ms}$ & $79 \mathrm{~ms}$ \\
Preserving patch's face & $1016 \mathrm{~ms}$ & $4454 \mathrm{~ms}$ & $89 \mathrm{~ms}$ \\
Using distance from border & $962 \mathrm{~ms}$ & $1050 \mathrm{~ms}$ & $90 \mathrm{~ms}$ \\
\hline
\end{tabular}

Table 1: Result table for experiment shown in Figure 9

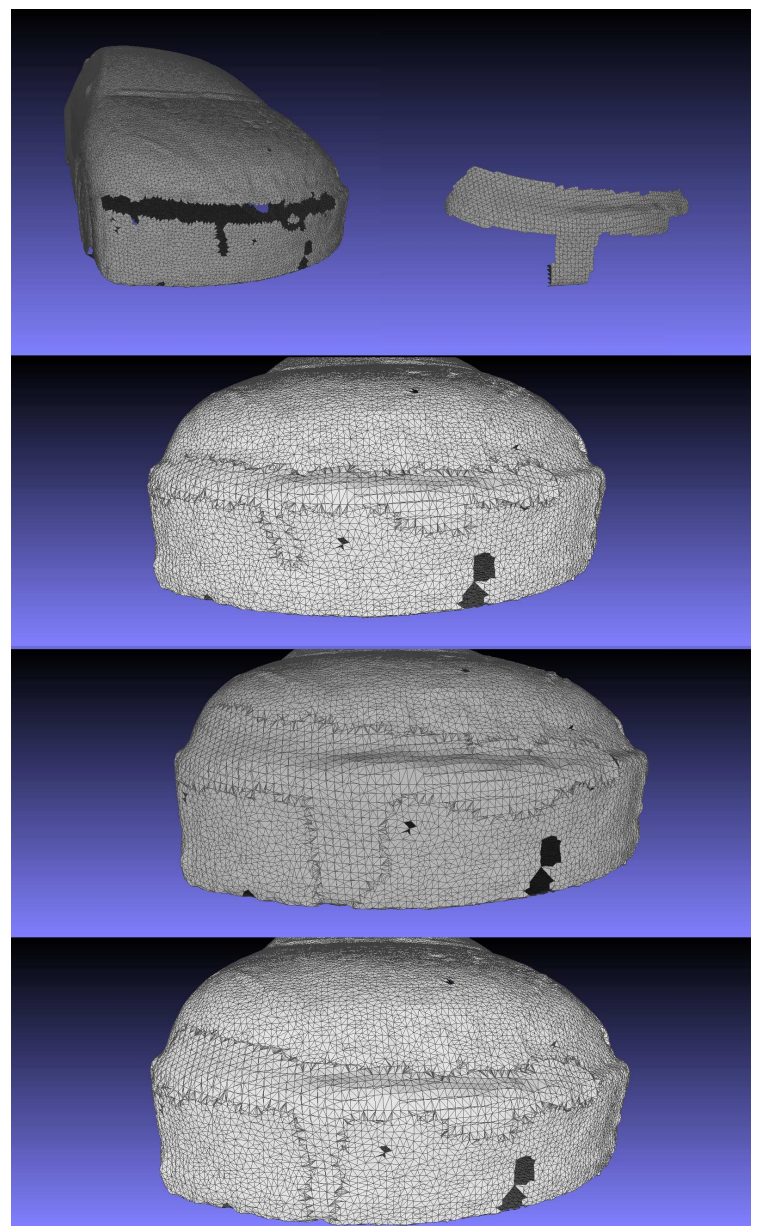

Figure 9: A large hole on the surface of a mesh and zippering with three different criteria: faces from mesh are preserved, then faces from patch are preserved and, finally, faces are weighted using their distance from border.

\section{REFERENCES}

Alexa, M., Behr, J., Cohen-Or, D., Fleishman, S., Levin, D., and Silva, C. T. (2001). Point set surfaces. In VIS '01: Proceedings of the conference on Visualization '01, pages 21-28, Washington, DC, USA. IEEE Computer Society.

Bernardini, F., Mittleman, J., Rushmeier, H., Silva, C., and Taubin, G. (1999). The ball-pivoting algorithm for surface reconstruction. IEEE Trans-

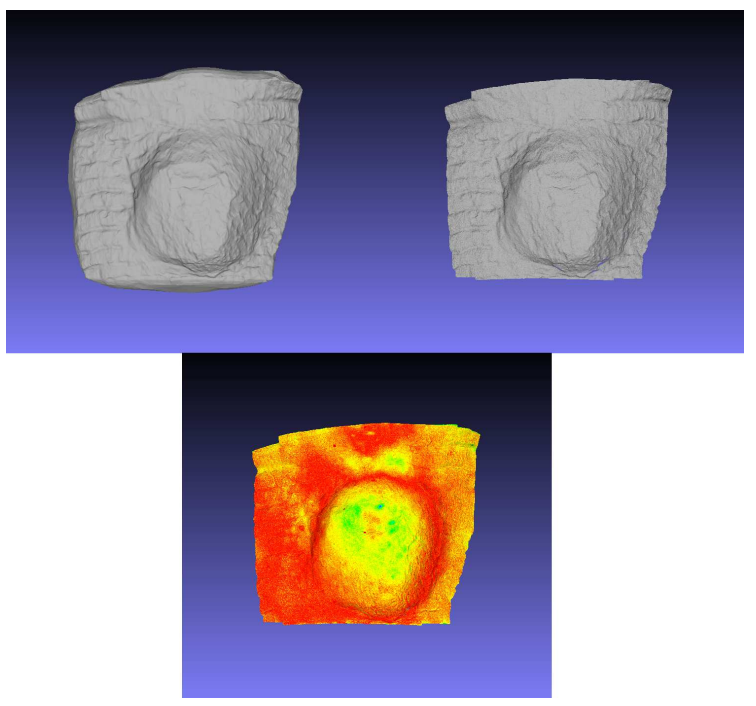

Figure 10: Poisson reconstruction, on the left, and zipperend range maps on the right. The bottom of the figure shows Hausdorff distance between the two results mapped to a ramp between red $(0 \mathrm{~m})$ and blue $(0.001 m)$.

actions on Visualization and Computer Graphics, 5:349-359.

Breuckmann. Breuckmann smartscan http://www . breuckmann. com.

Cignoni, P., Callieri, M., Corsini, M., Dellepiane, M., Ganovelli, F., and Ranzuglia, G. (2008). MeshLab: an Open-Source Mesh Processing Tool. In Sixth Eurographics Italian Chapter Conference, pages 129-136.

Curless, B. (1999). From range scans to 3D models. Computer Graphics, 33(4):38-41.

Dellepiane, M., Venturi, A., and Scopigno, R. (2009). Image guided reconstruction of un-sampled data: a coherent filling for uncomplete cultural heritage models. In IEEE Workshop on eHeritage and Digital Art Preservation, page In press. IEEE.

Duan, Y. and Qin, H. (2003). 2.5 d active contour for surface reconstruction. Proceedings of the Eighth International Workshop on Vision, Modeling, and Visualization (VMV 2003), page 431439.

Edelsbrunner, H. and Mücke, E. P. (1994). Three- 
Dimensional Alpha Shapes. ACM Transactions on Graphics, 13:43-72.

Fiorin, V., Cignoni, P., and Scopigno, R. (2007). Outof-core MLS reconstruction. In Proc. of he Ninth IASTED International Conference on Computer Graphics and Imaging (CGIM), pages 27-34.

Guennebaud, G. and Gross, M. (2007). Algebraic point set surfaces. ACM Transactions on Graphics, 26:23.

Hoppe, H., DeRose, T., Duchamp, T., McDonald, J., and Stuelzle, W. (1992). Surface reconstruction from unorganized points. In ACM Computer Graphics Proc., Annual Conference Series, (SIGGRAPH 92), pages 71-78.

Kazhdan, M., Bolitho, M., and Hoppe, H. (2006). Poisson surface reconstruction. Proceedings of the fourth Eurographics symposium on Geometry processing, page 70 .

NextEngine. Nextengine laser scanners. http://www. nextengine.com/.

Ohtake, Y., Belyaev, A., Alexa, M., Turk, G., and Seidel, H.-P. (2003). Multi-level partition of unity implicits. ACM Transactions on Graphics, 22:463-470.

Pereira, M. G., Anderson, S., Davis, J., Ginsberg, J., Shade, J., and Fulk, D. (2000). The Digital Michelangelo Project: 3D Scanning of Large Statues. Proc. ACM SIGGRAPH Conf., pp:131144.

Shen, C., O'Brien, J. F., and Shewchuk, J. R. (2005). Interpolating and approximating implicit surfaces from polygon soup. ACM SIGGRAPH 2005 Courses on - SIGGRAPH '05, page 204.

Turk, G. and Levoy, M. (1994). Zippered polygon meshes from range images. ACM Computer Graphics, 28:311-318. 